\title{
Curcumin-loaded nanostructured lipid carrier induced apoptosis in human HepG2 cells through activation of the DR5/caspase-mediated extrinsic apoptosis pathway
}

\author{
FENGLING WANG ${ }^{1,2}$ \\ XI YE ${ }^{1}$ \\ DANDAN ZHAI ${ }^{1}$ \\ WENTING DAI \\ YIFAN WU ${ }^{2}$ \\ JIN CHEN $^{1}$ \\ WEIDONG CHEN ${ }^{2, *}$ \\ ${ }^{1}$ Department of Pharmacy \\ The Second People's Hospital of Hefei \\ Hefei 230011, Anhui, China \\ ${ }^{2}$ Institute of Drug Metabolism \\ School of Pharmaceutical Sciences \\ Anhui University of Chinese Medicine \\ Hefei 230012, Anhui, China
}

Accepted April 19, 2019

Published online May 16, 2019

\begin{abstract}
Curcumin is a lipophilic anti-cancer compound extracted from turmeric. Our previous study demonstrated that the curcumin-loaded nanostructured lipid carrier (Cur-NLC) exhibits superior anti-cancer activity in inhibiting proliferation as well as inducing apoptosis of human HepG2 cells compared to native curcumin. This study aims to unveil the mechanisms underlying the pro-apoptotic effect of CurNLC on HepG2 cells. Evidence indicates that low expression of death receptors (DRs) on cancer cell membranes leads to attenuated apoptosis signaling. This study showed that CurNLC significantly increased total expression of DR5 protein while simultaneously upregulated cell membrane expression of DR5. Cur-NLC significantly increased caspase-8 and caspase-3 activities, accompanied by increased apoptosis. Furthermore, enhanced apoptosis was inhibited in the presence of a pan-caspase inhibitor, Z-VAD-FMK. Therefore, Cur-NLC induced activation of the extrinsic apoptosis pathway via modulating the DR5/caspase-8/-3 mediated apoptosis pathway in HepG2 cells, suggesting that Cur-NLC is a promising therapeutic agent or supplement for the treatment of hepatocellular carcinoma.
\end{abstract}

Keywords: curcumin-loaded nanostructured lipid carrier, apoptosis, death receptor 5, caspase-8, caspase-3, HepG2 cells

Surgical resection, liver transplant and chemotherapy currently act as main treatment options to control hepatocellular carcinoma (HCC), the prevailing primary malignancy of the liver (1). Nevertheless, the efficacy of several existing targeted therapies is often limited by the complicated pathogenesis of HCC and numerous unexpected effects of chemotherapeutics, including drug resistance and unavoidable toxicity at effective doses (2). Moreover, unprecedented high cost also restricts clinical application of chemotherapeutic drugs. Thus, novel and effective alternative therapies with lower toxicity and improved targeting for HCC management are urgently needed. Herbal medicinal products have at-

*Correspondence; e-mail: wdchen@ahtcm.edu.cn 
tracted growing attention as one of the best approaches for the discovery of new chemotherapeutic molecules due to their multi-target, multi-way, and low cytotoxicity profiles (3). To date, a large body of herbal medicine-derived compounds has been explored and reported to show anti-cancer activity and prevent the angiogenesis and metastasis of HCC (3). Therefore, curcumin, derived from the rhizome of Curcuma longa L. (commonly known as turmeric), was chosen for this investigation. It is a predominant constituent of curcuminoids and has been used as a golden spice and coloring agent for a long time in food industry, or for centuries for its medicinal benefits mainly in Asian countries including India and China (4). Clinical studies have revealed that curcumin possesses anti-cancer activity by suppressing tumor initiation, promotion and metastasis alone or in combination with other anti-cancer drugs (5). The anti-cancer activity of curcumin is probably attributed to its pleiotropic effects; it suppresses cancer cell proliferation and induces apoptosis in cancer cells (6). Notably, direct evidence from clinical trials indicates that curcumin works well and has excellent tolerability of high doses with very low toxicity, which is highly attractive and exciting for the therapeutic use of curcumin (7).

Despite its promise, curcumin as a lipophilic polyphenol has poor gastrointestinal absorbability and low systemic bioavailability in humans (8). Hence, the largest barrier for curcumin in cancer related therapy is to realize its full potential therapeutic efficacy. To circumvent these problems, the effectiveness of nanoparticulated curcumin has been tested against a number of cancer cell lines including HCC (9). Data showed nanoparticulated curcumin not only apparently improved the anti-cancer efficacy of curcumin but also increased its bioavailability compared to an identical amount of native curcumin (9). We have recently developed a curcumin-loaded nanostructured lipid carrier (Cur-NLC) that not only considerably enhanced the systemic bioavailability of this agent but also improved its anti-cancer activity in human lung A549 cells via enhancing cellular uptake and internalization of native curcumin (10). Previous data from our group revealed that CurNLC apparently increased hepatocarcinoma cell death in a concentration-dependent manner compared to native curcumin (11); however, the possible mechanisms underlying the induction of cell death have not been elucidated. It is therefore particularly interesting to understand the potential anti-cancer activity of Cur-NLC against human HepG2 hepatocarcinoma cells.

Induction of cell death is the main approach to eradicating cancer cells from the body. One of the major forms of cell death is apoptosis, which can be a powerful tool in the chemotherapy for cancer. Decrease of apoptosis is involved in both tumorigenesis and chemoresistance, which are the most common causes of treatment failures (12). A group of cysteine proteases called caspases are triggered by either intrinsic (mitochondrion-mediated) or extrinsic [(death receptor (DR)-mediated] apoptosis pathways. Extrinsic apoptotic pathway is initiated on the cell surface by ligation of a ligand to death receptors 4 (DR4) and DR5. Ligated DR promotes the formation of a death-inducing signaling complex (DISC) at the plasma membrane and subsequently transmits apoptosis signals via recruitment of adaptor molecules such as caspase- 8 and caspase-10, leading to the activation of caspase- 3 and induction of cellular apoptosis (13). Both DR4 and DR5 contain a conserved cytoplasmic region that is referred to as 'death domain', which is required for tumor-necrosis-factor-related apoptosis-inducing ligand (TRAIL)-induced apoptosis. Since DR4 and DR5induced apoptosis has been investigated against cancers, a better understanding of the role and mechanism/s of Cur-NLC mediated regulation of DR4 and DR5-induced apoptosis 
may result in a novel therapeutic opportunity against HCC. To the best of our knowledge and belief, this is the first study demonstrating the role of DRs/caspases-mediated extrinsic apoptosis pathways involved in Cur-NLC induced HepG2 cell apoptosis.

\section{EXPERIMENTAL}

\section{Materials}

Curcumin (purity $>98 \%$ ) and Tween-80 were purchased from Sinopharm Chemical Reagent Co., Ltd. (China). Lecithin and octyl decyl acid triglycerate were provided by Anhui Fengyuan Pharmaceutical Co., Ltd. (China). Poloxamer 188 (Pluronic F68) was purchased from BASF (Germany). Monostearin (MS) was obtained from Hunan Er-Kang Pharmaceutical Co., Ltd. (China). HPLC grade methanol was purchased from Merck (Germany). RPMI-1640 medium, defined fetal bovine serum (FBS), dimethyl sulfoxide (DMSO) and $1 \%$ penicillin-streptomycin were obtained from Gibco Co. (USA). DR4 (D-6, sc-166624), DR5 (B-9, sc-8411) and $\beta$-actin (C4, sc-47778) primary antibodies were obtained from Santa Cruz Biotechnology (USA). Secondary horseradish peroxide (HRP)-conjugated anti-mouse IgG antibody was obtained from Boster Bio-engineering Limited Company (China). RIPA lysis buffer and BCA protein assay kit were from Beyotime Biotechnology (China). Annexin V-fluorescein isothiocyanate/propidium iodide (Annexin V-FITC/PI) was purchased from Nanjing Keygen Biotech Co., Ltd. (China). Caspase -8, -10, and -3 colorimetric Assay Kits (BB-4107-2/BB-4112-2/BB-4106-2) were obtained from Shanghai BestBio Co., Ltd. (China). Enhanced chemiluminescence (ECL) reagent was bought from Pierce (USA). Caspase inhibitor Z-VAD-FMK (S7023) was purchased from Selleck.cn (China). Deionized water was provided by a Milli-Q Reference Ultrapure Water System, Merck (Germany). All other chemicals used in the experiment were of analytical grade.

\section{Preparation of Cur-NLC}

Curcumin-loaded solid lipid nanoparticles (Cur-SLN) were formulated as previously reported by our group (14). An NLC is a modified SLN with much more disordered and less crystalline lipid matrix, which can considerably improve the loading capacity and release properties, decrease drug leakage and increase chemical stability of incorporated drugs. Based on the formulation procedure of Cur-SLN, Cur-NLC was formulated by adding an additional liquid lipid (octyl decyl acid triglycerate). In brief, the lipid phase and the lipophilic surfactant were dissolved in moderate amounts of organic solvents and a homogeneous solution was obtained when heated up to $75^{\circ} \mathrm{C}$. After adding curcumin, the lipid phase was poured into the aqueous solution of hydrophilic surfactant and heated up to $75^{\circ} \mathrm{C}$, accompanied by high speed stirring $(1000 \mathrm{rpm})$ for $2 \mathrm{~h}$ to form a pre-emulsion. The resulting hot o/w nano-emulsion was dispersed into ice-cold distilled water immediately under gentle mechanical stirring for $2 \mathrm{~h}$. Cur-NLC was formed by lipid recrystallization. Orthogonal tests were conducted to optimize the composition of the solid lipid, liquid lipid and the surfactants. Using photon correlation spectroscopy with Zetasizer (Nano ZS90, Malvern, UK), the physicochemical characteristics including mean particle size, polydispersity index (PDI) and Zeta potential were determined after appropriate dilution with deionized water. The encapsulation efficiency $(E E)$ and drug loading $(D L)$ of Cur-NLC were assessed indirectly by HPLC analysis based on the following equation: 
and

$$
E E \%=\left(m_{\text {total }}-m_{\text {free }}\right) / m_{\text {total }} \times 100 \%
$$

$$
D L \%=\left(m_{\text {total }}-m_{\text {free }}\right) /\left(m_{\text {total }}+m_{\text {Lipid }}\right) \times 100 \%,
$$

where $m_{\text {total }}, m_{\text {free }}$ were the weight of total drug in the system and weight of free drug in the filtrate, respectively; and $m_{\text {Lipid }}$ was the weight of lipid material. The mean particle diameter, zeta potential, PDI, EE and DL of Cur-NLC were $99.99 \pm 1.87 \mathrm{~nm},-19.9 \pm 0.65 \mathrm{mV}, 0.158 \pm 0.005$, $(97.86 \pm 0.72) \%$, and $(4.35 \pm 0.12) \%$, respectively. Empty NLC were prepared by the same procedure without adding curcumin.

\section{Human HepG2 cell culture}

Human HepG2 cell line was obtained from the Shanghai Cell Bank, Chinese Academy of Sciences (China). HepG2 cell was incubated in the RPMI-1640 medium with $10 \%$ fetal bovine serum containing $100 \mathrm{U} \mathrm{mL}^{-1}$ penicillin and $100 \mu \mathrm{g} \mathrm{mL} \mathrm{L}^{-1}$ streptomycin at $37^{\circ} \mathrm{C}$ and $5 \% \mathrm{CO}_{2}$. Cells in the exponential growth phase were used for experiments. Cur-NLC and empty NLC were prepared as $80 \mathrm{mg} \mathrm{L}^{-1}$ stock solutions stored at $-20^{\circ} \mathrm{C}$ and diluted to different working solutions with medium before the treatment. After $24 \mathrm{~h}$, the cells were collected and stored at $-20^{\circ} \mathrm{C}$ for different analyses as described below.

\section{Determination of DR4 and DR5 proteins by Western blot analysis}

Cells were collected, washed with ice-cold PBS and lysed at a density of $0.5 \times 10^{6}$ cells $\mathrm{mL}^{-1}$ of RIPA lysis buffer containing $10 \mathrm{mM}$ phenylmethylsulfonyl fluoride (PMSF) and 0.1 $\%$ sodium dodecyl sulfate (SDS) for $30 \mathrm{~min}$ on ice. The BCA protein assay was used for measuring protein concentration according to the recommended guidelines. One protein sample was mixed with $5 \times$ sample buffer (4:1) and denatured at $95^{\circ} \mathrm{C}$ for $10 \mathrm{~min}$. The proteins were resolved by SDS-PAGE and then transferred onto a polyvinylidene difluoride membrane (Millipore, Bedford, MA) in transfer buffer ( $25 \mathrm{mM}$ Tris-base, $192 \mathrm{mM}$ glycine, and $20 \%$ methanol) at $4{ }^{\circ} \mathrm{C}$ for $2 \mathrm{~h}$. After blocking with $5 \%$ non-fat dry milk dissolved in $0.05 \%$ Tween 20-PBS, the membranes were incubated with the primary mouse anti-DR4 and anti-DR5 antibody under gentle agitation at $4{ }^{\circ} \mathrm{C}$ for $24 \mathrm{~h}$. After washing 3 times with PBS, the membranes were probed with an HRP-conjugated anti-mouse IgG antibody. Immunodetection was performed using ECL reagent according to the manufacturer's protocols. Equivalent protein loading and transfer efficiency were checked by staining for $\beta$-actin (a housekeeping gene). Control groups included cells treated only with complete growth culture medium (control) and cells treated with empty NLC (NLC control). All the experiments were performed in triplicate. The relative intensity of protein expression was normalized to $\beta$-actin. Autoradiographs were scanned using an Alpha Chemiluminescent gel imaging system FluorChem FC3 (ProteinSimple, Silicon Valley, USA) and quantified using ImageJ software (version 1.45S, NIH, USA).

\section{Determination of cell membrane proteins DR4 and DR5 by flow cytometry analysis}

Surface expression of membrane proteins DR4 and DR5 was quantified using the immunofluorescence labeling assay. Cells $\left(2 \times 10^{6}\right.$ cells $\left.\mathrm{mL}^{-1}\right)$ were washed twice with cold PBS and fixed with $4 \%$ paraformaldehyde at room temperature for $40 \mathrm{~min}$. After centrifugation at $1000 \mathrm{rpm}$ for $5 \mathrm{~min}$, the supernatant was discarded and cells were re-suspended in PBS, centrifuged at $1000 \mathrm{rpm}$ for $5 \mathrm{~min}$, and permeabilized with $0.1 \%$ Triton X-100 in PBS. Samples were blocked with $0.5 \%$ normal bovine serum albumin for $30 \mathrm{~min}$ and in- 
cubated with the primary antibodies at $4{ }^{\circ} \mathrm{C}$ overnight. After washing 3 times, cells were probed with a secondary fluorescein isothiocyanate (FITC)-conjugated antimouse IgG antibody and kept in a dark place for $1 \mathrm{~h}$ at $4{ }^{\circ} \mathrm{C}$. After staining, cells were washed again with ice-cold PBS and re-suspended in PBS. $200 \mu \mathrm{L}$ cell samples were transferred to $5 \mathrm{~mL}$ Falcon tubes and the samples were analyzed using a Flow Cytometer (USA). NLC control, model control (without primary antibody and secondary FITC-conjugated anti-mouse IgG antibody) and isotype (without primary antibody) groups were used as controls. Experiments were performed in triplicate; data compensation and the mean fluorescence intensity from different samples were performed using the FlowJo Version 7.6.1 software (USA).

\section{Apoptosis analysis by flow cytometry}

To confirm whether caspases participate in Cur-NLC induced HepG2 cell apoptosis, the effect of Cur-NLC combined with caspase inhibitor (Z-VAD-FMK) on the HepG2 cell apoptosis rate was analyzed by flow cytometry. HepG2 cells were stained with an Annexin V-FITC/PI apoptosis detection kit that detects phosphatidylserine exposed to the outer surface of the cell membrane for fluorescence-activated cell sorting (FACS) analysis. In brief, HepG2 cells $\left(0.5 \times 10^{6}\right.$ cells per well $)$ were added into a 6 -well plate and cultured until 75 85\% confluence. Cells were then treated with Cur-NLC $\left(10 \mu \mathrm{mol} \mathrm{L}^{-1}\right)$ and CurNLC $\left(10 \mu \mathrm{mol} \mathrm{L}^{-1}\right)$ combined with Z-VAD-FMK $\left(20 \mu \mathrm{mol} \mathrm{L}^{-1}\right)$, respectively. The model group (cells cultured with complete growth culture medium only) and the NLC control group acted as controls. After $48 \mathrm{~h}$, cells were collected by trypsinization and washed 3 times with cold PBS and centrifuged at $1000 \mathrm{rpm}$ for $5 \mathrm{~min}$. Cells were stained by adding Annexin V-FITC/PI into binding buffer. Stained cells were put in a dark place at $37^{\circ} \mathrm{C}$ for $15 \mathrm{~min}$. Processed single cell suspensions were analyzed with a flow cytometer. Triplicate of each sample was examined and data compensation was performed using FlowJo Version 7.6.1 software. It should be noted that cells staining negative for both annexin $\mathrm{V}$ and PI are live cells, cells labelled with annexin $\mathrm{V}^{+} / \mathrm{PI}^{-}$are in early apoptosis phase, and cells labelled with annexin $\mathrm{V}^{+} / \mathrm{PI}^{+}$are in the necrotic or late apoptosis phase.

\section{Measurement of Caspase-8, -10,-3 activation}

To measure caspase activity, caspase- $8,-10$, and -3 , colorimetric assay kits were used according to the manufacturer's instructions. The assay was based on the spectrophotometric detection of chromophore p-nitroanilide (pNA) after cleavage from the labeled substrate DEVD-pNA. The cells $\left(1 \times 10^{6}\right)$ were harvested, lysed and incubated on ice for $30 \mathrm{~min}$. Protein lysate was collected and quantified. Protein samples $(100 \mu \mathrm{g})$ were placed into a 96-well plate and mixed respectively with $4 \mathrm{mmol} \mathrm{L}^{-1}$ DEVD-pNA (caspase-3), IETD-pNA (caspase-8) and AEVD-pNA (caspase-10). The mixtures were then incubated at $37^{\circ} \mathrm{C}$ for $2 \mathrm{~h}$ in the dark and then the activity was measured at $405 \mathrm{~nm}$ using a microplate reader (BioTek Elx $\times 800$ microplate reader, Winooski, VT, USA). The experiment was performed in triplicate. Comparison of the absorbance of p-NA from an apoptotic sample with an uninduced control allows determination of the fold increase in caspase activity.

\section{Statistical analysis}

Each experiment was conducted in triplicate and the values were expressed as mean \pm standard deviations (SD). Biostatistical analyses were assessed using GraphPad Prism version 5.0 for Windows (GraphPad software, San Diego California USA, www.graphpad. 
com). Statistical comparisons of multiple groups were performed with one-way ANOVA followed by the Student-Newman-Keuls post hoc test and $p<0.05$ was considered as statistically significant.

\section{RESULTS AND DISCUSSION}

\section{Cur-NLC increased the expression of DR5 protein in HepG2 cells}

DR4 and DR5, as death receptors on the surface of cells, can promote one of the apoptotic pathways, that is, the extrinsic pathway, through DISC assembly and subsequent activation of caspases. Therefore, any change concerning the protein expression level of the two death receptors exerts a profound influence on apoptosis signaling. Hence, aberrant cell surface expression of DR4 and DR5 might play a critical role in the death-inducing ability. In this regard, surface expression of DR4 and DR5 could be evaluated as a predictive biomarker for identifying patient subpopulations with tumors that are responsive or resistant to death receptor targeted agents.

It is worth noting that DR5 may make a greater contribution than DR4 to TRAIL-induced apoptosis in cancer cells (which express both death receptors). The present study

a)

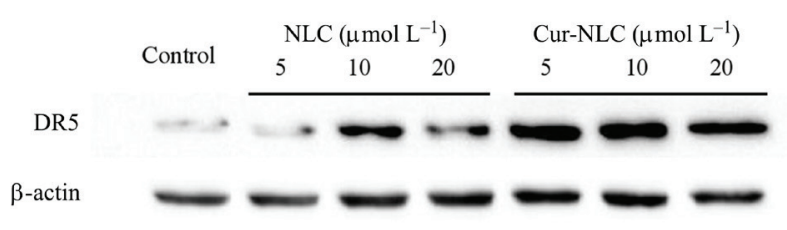

b)

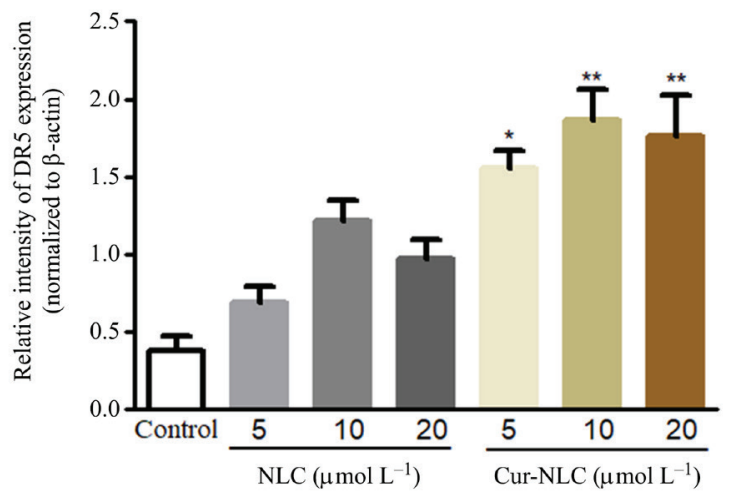

Fig. 1. Effect of Cur-NLC on the total protein expression of death receptor 5 (DR5) in human HepG2 cells $(x \pm s, n=3)$ : a) Total protein expression of DR5 in HepG2 cells assessed by Western blot analysis; b) Bar graphs represent quantitative evaluation of DR5 bands by densitometry from triplicate independent experiments. Representative image of the immunoblot for DR5 is shown. Relative protein level of DR5 was analyzed using $\beta$-Actin as a loading control. ${ }^{*} p<0.05,{ }^{* *} p<0.01$ compared to the control group (non-treated cells). 
measured the total and cell surface protein expression of DR4 and DR5 after Cur-NLC treatment. To investigate whether Cur-NLC has anti-cancer ability against HepG2 cells, we treated HepG2 cells with Cur-NLC at doses of 5, 10 and $20 \mu \mathrm{mol} \mathrm{L}^{-1}$ for $48 \mathrm{~h}$ based on our previous results (11). As shown in Fig. 1, HepG2 cells treated with Cur-NLC resulted in upregulation of total DR5 protein expression compared to control cells. It seems that the expression of DR5 in NLC groups was higher than that of the control group, but it did not reach a significant difference. Furthermore, to determine whether Cur-NLC increased the protein expression of DR5 on cell surface, the cells were subjected to an immunofluorescence labeling assay. Flow cytometry analysis showed that the protein expression of cell membrane DR5 increased in a concentration-dependent manner after treatment with CurNLC compared to the control group (Fig. 2), which is consistent with the result of Western blot. No significant changes in total and membrane DR4 protein expression were observed

a)

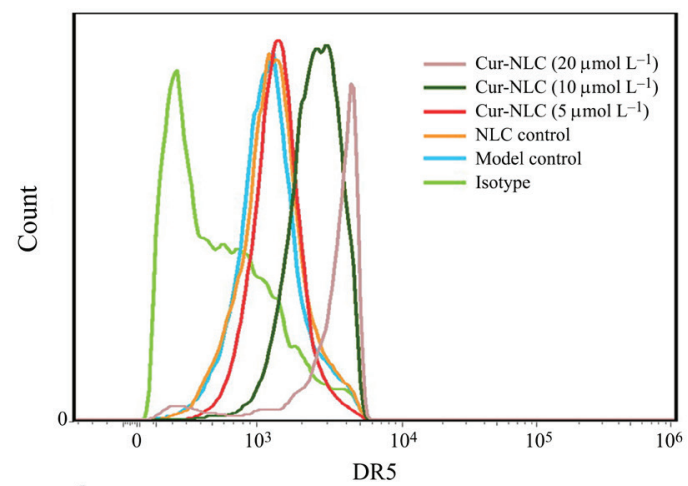

b)

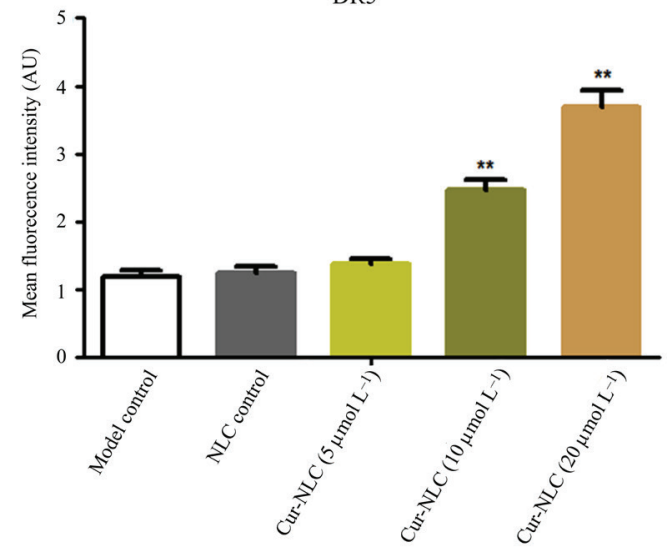

Fig. 2. Effect of Cur-NLC on the translocation of death receptor 5 (DR5) to the plasma membrane surface $(x \pm s, n=6)$ : a) Membrane protein expression of DR5 in HepG2 cells examined by flow cytometric analysis; $b$ ) Bar graphs show quantitative evaluation of DR5 bands from triplicate independent experiments by the biological image analysis system. Model group represents cells cultured with complete growth culture medium only, and NLC group represents cells treated with NLC without curcumin in the same concentration as Cur-NLC group $\left(10 \mu \mathrm{mol} \mathrm{L}^{-1}\right)$.

${ }^{* *} p<0.01$ compared to the model control group 
after treatment with Cur-NLC (Supplementary Fig. S1 and S2). The data indicated that CurNLC increased the expression of DR5, but not DR4 in human HepG2 cells (Fig. 1). Hence, therapeutic drugs that can upregulate DR5 protein expression may be likely to increase cellular sensitivity to apoptosis therapies.

\section{Caspases activation is involved in Cur-NLC induced apoptosis}

Similar results for protein expression of total DR5 and membrane DR5 indicate that Cur-NLC could significantly induce enhanced DR5 expression in HepG2 cells. In general, DR5-dependent apoptosis involves a cascade of events, such as activation of caspases, which is commonly considered to have a predominant role in apoptosis signaling pathways. To further evaluate the involvement of the extrinsic pathway apoptosis of HepG2 cells induced by Cur-NLC, the effects of Cur-NLC combined with the pan-caspase inhibitor (Z-VAD-FMK) on the HepG2 cell apoptosis rate were assessed. HepG2 cells were pretreated with Z-VAD-FMK for $2 \mathrm{~h}$ before Cur-NLC treatment. Flow cytometry was used to detect the apoptosis rate of treated cells. As shown in Fig. 3, an apparent increase in the percentage of HepG2 cell apoptosis was observed immediately after Cur-NLC $(10 \mu \mathrm{mol}$ $\mathrm{L}^{-1}$ ) treatment compared to the control. However, a significant decrease of Cur-NLC induced apoptosis was found in the presence of Z-VAD-FMK $\left(20 \mu \mathrm{mol} \mathrm{L}^{-1}\right)$ compared to the

a)
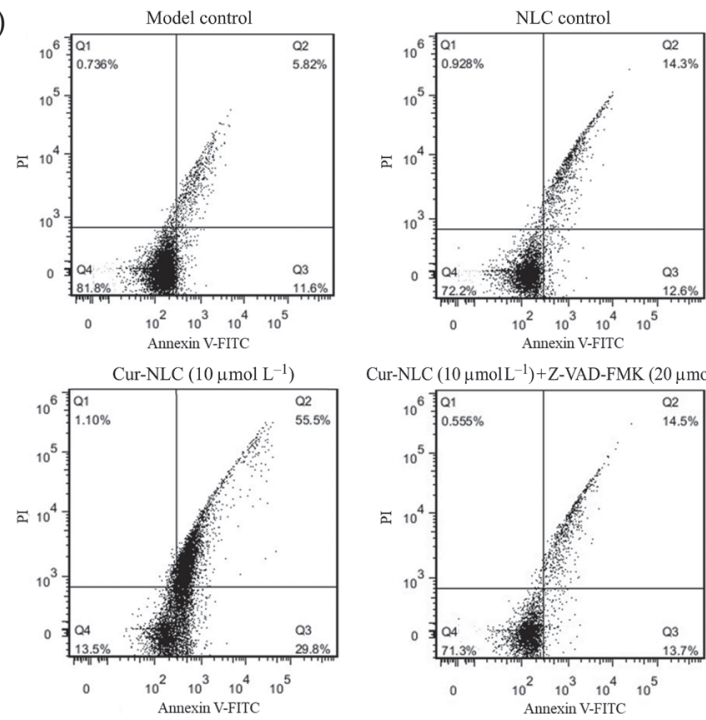

Cur-NLC $\left(10 \mu\right.$ molL $\left.^{-1}\right)+$ Z-VAD-FMK $\left(20 \mu\right.$ mol L $\left.^{-1}\right)$

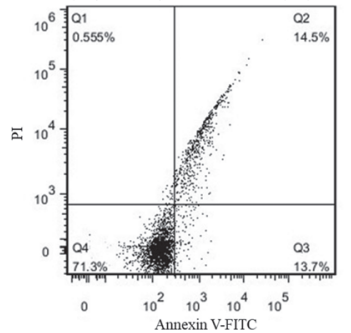

b)

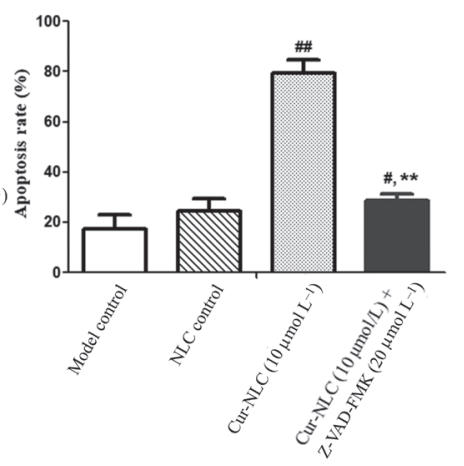

Fig. 3. Effects of Cur-NLC and the pan-caspase inhibitor (Z-VAD-FMK) on HepG2 cell apoptosis rate $(x \pm s, n=3)$ : a) Apoptotic cells detected by flow cytometry. Cells were treated with Cur-NLC $\left(10 \mu \mathrm{mol} \mathrm{L}^{-1}\right)$ and pre-treated with Z-VAD-FMK at $20 \mu \mathrm{mol} \mathrm{L}^{-1}$ for $2 \mathrm{~h}$ before Cur-NLC treatment. Model group represents cells cultured with complete growth culture medium only, and NLC group represents cells treated with NLC without curcumin in the same concentration as Cur-NLC group $\left.(10 \mu \mathrm{mol} \mathrm{L})^{-1}\right)$. The lower right quadrant (annexin $\mathrm{V}^{+} / \mathrm{PI}^{-}$) represents early apoptosis, while the upper right quadrant (annexin $\mathrm{V}^{+} / \mathrm{PI}^{+}$) represents late apoptosis or necrosis; b) Bar graphs represent the apoptosis rate of each group analyzed from triplicate independent experiments by GraphPad prism 5 software. ${ }^{*} p<0.05$, ${ }^{\# \#} p<0.01$ vs. model control group; ${ }^{* *} p<0.01$ vs. Cur-NLC group. 

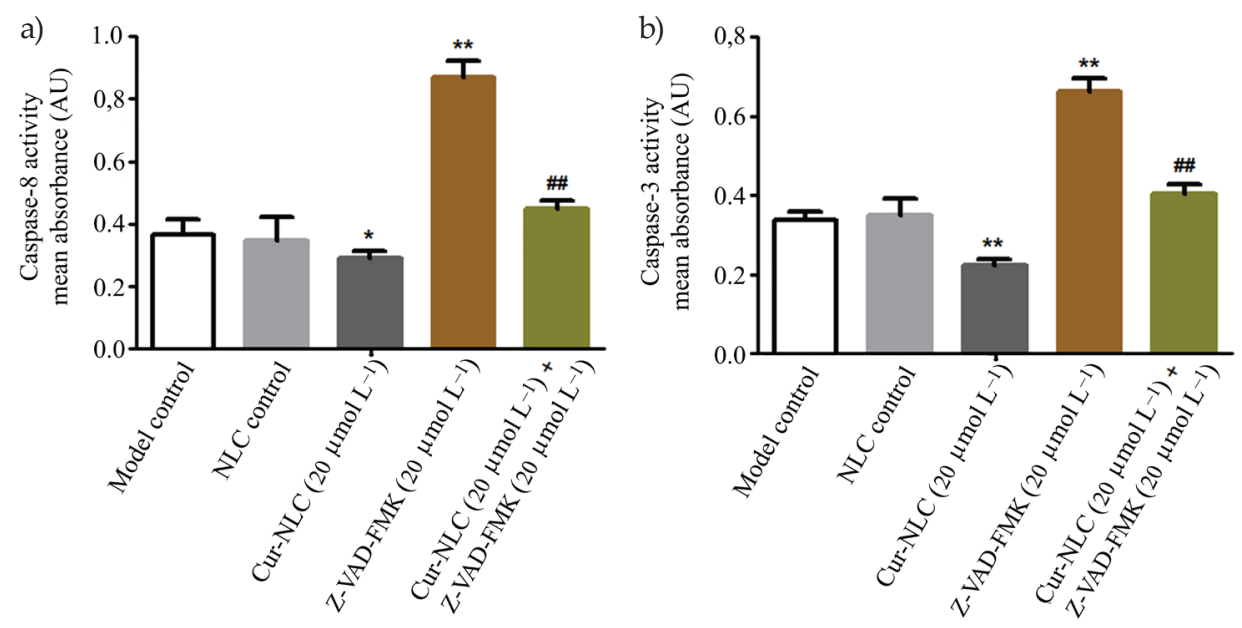

Fig. 4. Effects of Cur-NLC on caspase-8 and caspase-3 activities in HepG2 cells: a) Caspase-8 activation was determined by spectrophotometric detection of their cleavages by caspase- 8 colorimetric assay kits $(x \pm s, n=3)$; b) Caspase-3 activation was determined by spectrophotometric detection of their cleavages by caspase-3 colorimetric assay kits. Model group represents cells cultured with complete growth culture medium only, and NLC group represents cells treated with NLC without curcumin in the same concentration as Cur-NLC group $\left(20 \mu \mathrm{mol} \mathrm{L}^{-1}\right) .{ }^{*} p<0.05,{ }^{* *} p<0.01$ vs model control group; ${ }^{*} p<0.05,{ }^{* \sharp} p<0.01$ vs. Cur-NLC group.

cells treated with Cur-NLC alone. These results indicate that caspase activation is clearly associated with Cur-NLC induced apoptosis.

\section{Effect of Cur-NLC on caspase-8, -10 and -3 activity}

Within the extrinsic apoptotic pathway, caspase- 8 is the most proximal caspase that transmits apoptotic signals originating at the membrane of DRs (15). In order to investigate the possible mechanism underlying caspases-mediated apoptotic events, the effects of coadministration of Cur-NLC with caspase inhibitor on the activation of caspase-8, -10 and -3 were evaluated. Following Cur-NLC treatment, the activity of caspase- 8 and -3 were remarkably increased in HepG2 cells compared to the control. However, co-treatment of Cur-NLC with Z-VAD-FMK strongly suppressed the activity of caspase- 8 and -3 (Fig. 4A and B). No significant change was found in the activity of caspase-10 after treatment with Cur-NLC (Supplementary Fig. S3).

Taken together, these data suggest that Cur-NLC-induced HepG2 cell apoptosis may be mediated via an extrinsic death receptor pathway mechanism, i.e., through DR5 upregulation and activation of caspase- 8 and caspase- 3 .

\section{CONCLUSIONS}

Curcumin widespread availability, superior safety profile, low cost and multiple cancer fighting functions make it a potent anti-cancer herbal extract. The novelty of this study lies in the finding that Cur-NLC modulated DR5/caspase-8/-3 mediated extrinsic apoptosis 
pathway involved in HepG2 cell apoptosis. Thus, our previous and present studies collectively suggest that Cur-NLC may be a potential therapeutic and preventative drug for HCC in increasing cellular sensitivity to apoptosis. However, more preclinical as well as clinical studies are necessary to unveil the mechanisms underlying the pro-apoptotic effect of Cur-NLC on HCC. For instance, it is necessary to elucidate how, by targeting appropriate signal transducers, the Cur-NLC mediated apoptotic pathway works in the different HCC cell lines and animal models. More exact experiments will be carried out to unveil detailed pro-apoptotic effects of Cur-NLC in the extrinsic death receptor pathway. The proposed in vivo and in vitro studies for the molecular basis would provide valuable insights to evaluate the applicability of curcumin as a chemopreventive and/or therapeutic agent for HCC.

Acknowledgements. - This research was supported by the National Natural Science Foundation of China (NO. 81773988), Natural Science Foundation of Anhui University of Chinese Medicine (2018zrzd04), and Anhui Provincial Department of Education: Program for Scientific Research Innovation Team in Colleges and Universities of Anhui Province (NO. 2016hz23).

\section{REFERENCES}

1. J. Bruix and M. Sherman, Management of hepatocellular carcinoma: An update, Hepatology 53 (2011) 1020-1022; https://doi.org/10.1002/hep.24199

2. U. Asghar and T. Meyer, Are there opportunities for chemotherapy in the treatment of hepatocellular cancer? J. Hepatol. 56 (2012) 686-695; https://doi.org/10.1016/j. jhep.2011.07.031

3. S. R. Chen, H.C. Qiu, Y. Hu, Y. Wang and Y. T. Wang, Herbal medicine offered as an initiative therapeutic option for the management of hepatocellular carcinoma, Phytother. Res. 30 (2016) 863877; https://doi.org/10.1002/ptr.5594

4. B. Kocaadam and N. Sanlier, Curcumin, an active component of turmeric (Curcuma longa), and its effects on health, Crit. Rev. Food Sci. Nutr. 57 (2017) 2889-2895; https://doi.org/ 10.1080/10408398. 2015.1077195

5. H. Mahammedi, E. Planchat, M. Pouget, X. Durando, H. Cure, L. Guy, I. Van-Praagh, L. Savareux, M. Atger, M. Bayet-Robert, E. Gadea, C. Abrial, E. Thivat, P. Chollet and J. C. Eymard, The new combination docetaxel, prednisone and curcumin in patients with castration-resistant prostate cancer: A Pilot Phase II Study, Oncology 90 (2016) 69-78; https://doi.org/ 10.1159/000441148

6. W. Z. Wang, L. Li, M. Y. Liu, X. B. Jin, J. W. Mao, Q. H. Pu, M. J. Meng, X. G. Chen and J. Y. Zhu, Curcumin induces FasL-related apoptosis through p38 activation in human hepatocellular carcinoma Huh7 cells, Life Sci. 92 (2013) 352-358; https://doi.org/ doi:10.1016/j.lfs.2013.01.013

7. M. I. James, C. Iwuji, G. Irving, A. Karmokar, J. A. Higgins, N. Griffin-Teal, A. Thomas, P. Greaves, H. Cai, S. R. Patel, B. Morgan, A. Dennison, M. Metcalfe, G. Garcea, D. M. Lloyd, D. P. Berry, W. P. Steward, L. M. Howells and K. Brown, Curcumin inhibits cancer stem cell phenotypes in ex vivo models of colorectal liver metastases, and is clinically safe and tolerable in combination with FOLFOX chemotherapy, Cancer Lett. 364 (2015) 135-141; https://doi.org/ 10.1016/j.canlet.2015.05.005

8. R. A. Sharma, S. A. Euden, S. L. Platton, D. N. Cooke, A. Shafayat, H. R. Hewitt, T. H. Marczylo, B. Morgan, D. Hemingway, S. M. Plummer, M. Pirmohamed, A. J. Gescher and W. P. Steward, Phase I clinical trial of oral curcumin: biomarkers of systemic activity and compliance, Clin. Cancer Res. 10 (2004) 6847-6854; https://doi.org/ 10.1158/1078-0432.CCR-04-0744 
9. D. Ghosh, S. T. Choudhury, S. Ghosh, A. K. Mandal, S. Sarkar, A. Ghosh, K. D. Saha and N. Das, Nanocapsulated curcumin: oral chemopreventive formulation against diethylnitrosamine induced hepatocellular carcinoma in rat, Chem. Biol. Int. 195 (2012) 206-214; https://doi.org/ 10.1016/j. cbi.2011.12.004

10. F. Wang, J. Chen, W. Dai, Z. He, D. Zhai and W. Chen, Pharmacokinetic studies and anticancer activity of curcumin-loaded nanostructured lipid carriers, Acta Pharm. 67 (2017) 597-605; https:// doi.org/ 10.1515/acph-2017-0021

11. F. Wang, Z. He, W. Dai, Q. Li, X. Liu, Z. Zhang, D. Zhai, J. Chen and W. Chen, The role of the vascular endothelial growth factor/vascular endothelial growth factor receptors axis mediated angiogenesis in curcumin-loaded nanostructured lipid carriers induced human HepG2 cells apoptosis, J. Cancer Res. Ther. 11 (2015) 597-605; https://doi.org/10.4103/0973-1482.159086

12. S. Fulda, Targeting apoptosis for anticancer therapy, Semin. Cancer Biol. 31 (2015) 84-88. https://doi. org/10.1016/j.semcancer.2014.05.002

13. F. C. Kischkel, D. A. Lawrence, A. Chuntharapai, P. Schow, K. J. Kim and A. Ashkenazi, Apo2L/ TRAIL-dependent recruitment of endogenous FADD and caspase- 8 to death receptors 4 and 5, Immunity 12 (2000) 611-620; https://doi.org/10.1016/s1074-7613(00)80212-5

14. J. Chen, W. T. Dai, Z. M. He, L. Gao, X. Huang, J. M. Gong, H. Y. Xing and W. D. Chen, Fabrication and evaluation of curcumin-loaded nanoparticles based on solid lipid as a new type of colloidal drug delivery system, Indian J. Pharm. Sci. 75 (2013) 178-184.

15. J. Li and J. Yuan, Caspases in apoptosis and beyond, Oncogene 27 (2008) 6194-6206. https://doi.org/ 10.1038/onc.2008.297 